\title{
ANGIOGRAFIA POR RESSONÂNCIA MAGNÉTICA: ASPECTOS TÉCNICOS DE UM MÉTODO DE ESTUDO VASCULAR NÃO-INVASIVO*
}

\author{
Rogério Pedreschi Caldana ${ }^{1}$, Alexandre Sérgio de Araújo Bezerra' ${ }^{1}$, Adriano Fleury \\ de Faria Soares ${ }^{2}$, Giuseppe D'Ippolito ${ }^{3}$
}

Resumo As primeiras técnicas de angiografia por ressonância magnética (angio-RM) utilizavam seqüências sensíveis ao fluxo sanguíneo para estabelecimento do contraste vascular. Há três técnicas fundamentadas neste princípio: contraste de fase ("phase-contrast"), TOF ("time-of-flight") e as técnicas de sangue escuro ("black blood"). Estas seqüências, de aquisição demorada, são mais suscetíveis a artefatos de movimento, perda de sinal em áreas de estenoses ou turbilhonamento de fluxo, e apresentam ainda baixa sensibilidade à detecção do fluxo lento. 0 uso do contraste paramagnético para estudos angiográficos pela ressonância magnética ofereceu um método simples, rápido e de excelente detalhamento vascular, baseando o contraste da imagem no realce do sinal vascular em oposição à supressão dos demais tecidos. Metodologias modernas que priorizam a obtenção do espaço $k$ central, responsável pelo contraste da imagem, e o aperfeiçoamento das técnicas de planejamento do intervalo temporal para aquisição dos dados foram fatores fundamentais para o aprimoramento técnico da angio-RM. O papel atual da angio-RM como ferramenta diagnóstica merece destaque na avaliação de anomalias anatômicas, estenoses, oclusões e complicações vasculares pós-cirúrgicas, principalmente nos casos de transplantes de órgãos. Suas principais vantagens estão na não utilização do contraste iodado ou radiação ionizante, rapidez e fácil execução, mínima invasividade e possibilidade de avaliar complementarmente o parênquima de órgãos adjacentes de interesse diagnóstico. Unitermos: Angiografia; Ressonância magnética; Gadolínio.

Abstract Magnetic resonance angiography: technical aspects of a noninvasive method for vascular study.

Early magnetic resonance angiography techniques used flow-sensitive sequences to obtain contrast of the vascular structures. Three techniques are based on this approach: phase-contrast, time-of-flight and black blood techniques. However, these acquisition methods are time-consuming and more susceptible to movement artifacts, flow-related artifacts and signal loss due to stenotic lesions. They also present low sensitivity for slow flow detection. The use of paramagnetic contrast medium in magnetic resonance angiography studies provides a simple and quick way of obtaining vascular enhancement. Image contrast is based on vascular enhancement in opposition to background suppression. Important factors for the technical improvement of gadolinium-enhanced magnetic resonance angiography include modern techniques of central k-space ordering that are responsible for high image contrast, and implementation of appropriate timing injection. Gadolinium-enhanced magnetic resonance angiography is a useful diagnostic tool for investigation of anatomical anomalies, stenotic and occlusive disease, and surgical complications, particularly in patients with organ transplants. Gadolinium-enhanced magnetic resonance angiography has the advantage of a noninvasive method since it does not require ionizing radiation or an iodine contrast medium, and allows the evaluation of parenchymatous structures for additional diagnostic information.

Key words: Angiography; Magnetic resonance imaging; Gadolinium.

\section{INTRODUÇÃO}

A angiografia por ressonância magnética (angio-RM) tem sido cada vez mais uti-

\footnotetext{
* Trabalho realizado no Departamento de Diagnóstico po Imagem da Universidade Federal de São Paulo/Escola Paulista de Medicina (DDI-Unifesp/EPM), São Paulo, SP.

1. Pós-graduandos do DDI-Unifesp/EPM.

2. Médico Radiologista do Complexo Hospitalar São Luiz.

3. Professor Adjunto do DDI-Unifesp/EPM.

Endereço para correspondência: Dr. Rogério Pedreschi Caldana. Rua Napoleão de Barros, 800 , Vila Clementino. São Paulo, SP, 04024-002. E-mail: rogercal@uol.com.br

Recebido para publicação em 14/5/2003. Aceito, após revisão, em 15/7/2003.
}

lizada como método de estudo vascular, principalmente pelas importantes vantagens de ser um método não-invasivo e que dispensa o uso do contraste iodado, potencialmente alérgeno e nefrotóxico. Ganhos tecnológicos recentes tornaram a moderna técnica de angio-RM tridimensional com contraste um procedimento altamente refinado, envolvendo grande número de variáveis e recursos tecnológicos em sua execução. Neste trabalho discutiremos alguns dos principais aspectos técnicos deste método de estudo vascular.

\section{MECANISMOS DE CONTRASTE VASCULAR NA ANGIO-RM}

A identificação das estruturas vasculares depende de técnicas que produzam contraste entre os vasos e os tecidos estacionários adjacentes.

As primeiras técnicas desenvolvidas para angio-RM utilizavam sequiências sensíveis ao fluxo sanguíneo para estabelecimento deste contraste, sendo baseadas nas propriedades de sinal do sangue em movimento e dispensando o uso de contraste 
endovenoso. Existem basicamente três técnicas fundamentadas nesse contexto: contraste de fase (CF) ("phase-contrast"), "time-of-flight" (TOF) e as técnicas de sangue escuro ("black blood") (1,2).

Nestas seqüiências a aquisição das imagens geralmente é mais demorada, com maior suscetibilidade a artefatos de movimento, além da limitação da área de abrangência pelo estudo, que é determinada pelo campo de visão, atingindo extensão máxima de 200 a $300 \mathrm{~mm}$ para cada seqüência isolada $^{(3)}$ (Figura 1). Outra importante desvantagem da técnica TOF é a saturação "em plano", que pode produzir imagens falsas de perda de sinal em vasos tortuosos, de fluxo lento ou cujo maior eixo coincide com o plano de decodificação da fase (Figura 2). Há, também, perda de sinal em áreas de estenoses ou turbilhonamento de fluxo, secundária principalmente à dispersão de fase intravoxel, e conseqüente perda de nitidez exatamente nos locais de maior interesse diagnóstico ${ }^{(2,4)}$. Estas técnicas apresentam, ainda, baixa sensibilidade à detecção do fluxo lento, gerada por queda da relação contraste-ruído ${ }^{(\mathbf{1})}$. As imagens obtidas pela técnica TOF são também suscetíveis a artefatos de segmentação ("em escada"), devido a imperfeições na sobreposição dos cortes contíguos (efeito ilustrado na Figura 11).

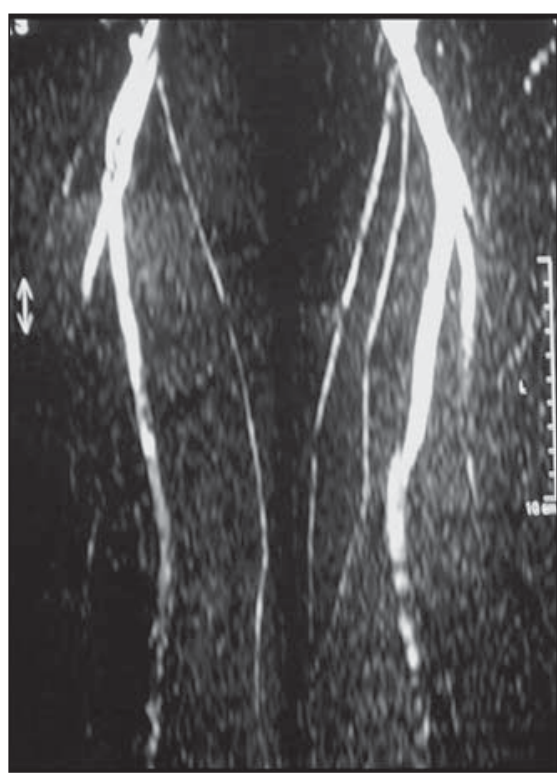

Figura 1. Exemplo de seqüência com técnica TOF direcionada ao estudo venoso dos membros inferiores. Notar o elevado nível de ruído da imagem.

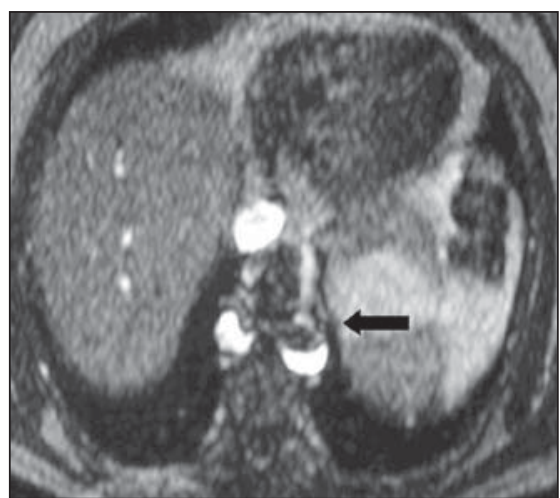

Figura 2. Seqüência no plano axial com técnica TOF demonstrando perda artefatual de sinal por efeito de saturação em plano, o que simula imagem de trombose em comunicação ectasiada do sistema ázigos/ hemi-ázigos (seta).

\section{ANGIO-RM COM GADOLÍNIO}

O uso do contraste paramagnético para estudos angiográficos pela RM foi introduzido na metade da década de $1990^{(\mathbf{5})}$. Devido à sua relativa simplicidade, boa qualidade de imagem e rapidez de aquisição, a angio-RM com gadolínio popularizou-se rapidamente, passando a ser aplicada nos diversos territórios vasculares e substituindo as técnicas previamente descritas, principalmente nos estudos extracranianos.

Esta técnica oferece excelente detalhamento vascular, e ao contrário das técnicas TOF e CF, como o sinal vascular não depende da detecção direta dos "spins" em movimento, efeitos de saturação por baixo fluxo e a perda de sinal induzida pela turbulência não constituem problema ${ }^{(6)}$. Atualmente, as sequiências TOF e CF são utilizadas como método complementar à angio-RM com contraste, como para o cálculo da velocidade de fluxo que pode ser obtida por $\mathrm{CF}^{(7)}$.

A angio-RM com gadolínio baseia-se no realce do sinal vascular promovido pela administração do contraste paramagnético, associado à pronunciada supressão dos tecidos estacionários, estabelecendo assim o alto contraste necessário para a imagem angiográfica $^{(\mathbf{4 , 8 , 9 )}}$ (Figura 3).

As seqüências gradiente-eco (GE) são especialmente úteis para essa finalidade, em que o uso de tempos de repetição extremamente baixos determina melhor supressão do sinal tecidual e maior velocidade de aquisição ${ }^{(\mathbf{1}, 10)}$. A aquisição mais rá-

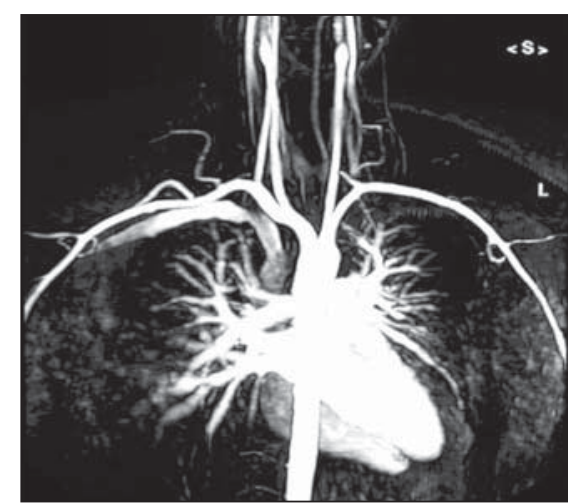

Figura 3. Angio-RM 3D com contraste do tórax apresentada na forma de reconstrução MIP no plano coronal. Notar o elevado contraste e o excelente detalhamento anatômico, em imagem comparável à arteriografia convencional.

pida torna possível a obtenção dos dados em formato volumétrico tridimensional, com grande benefício nas reconstruções de pós-processamento ${ }^{(\mathbf{1 1})}$.

A utilização do meio de contraste paramagnético (gadolínio) causa encurtamento do tempo de relaxamento T1 sanguíneo (de $1.200 \mathrm{~ms}$ para cerca de $100 \mathrm{~ms}$ ), evitando o efeito de supressão do sinal tecidual gerado pelo curto TR, e fornecendo excelente visualização das estruturas vasculares ${ }^{(\mathbf{1 2})}$. As informações obtidas são então manipuladas por programas de processamento para criar imagens angiográficas.

\section{TÉCNICA DE EXAME}

A técnica mais adequada para o estudo de angio-RM é baseada em sequiências tridimensionais GE ultra-rápidas, como a "fast field echo" (FFE). A redução do TR depende da potência ou capacidade dos gradientes disponíveis e intensidade do campo magnético. Nos equipamentos mais modernos, com gradientes eficientes, podem ser atingidos tempos de repetição de aproximadamente $3 \mathrm{~ms}^{(\mathbf{1})}$.

Estas seqüências permitem a aquisição das imagens em tempo inferior a 20 segundos, podendo ser completadas no período de uma única apnéia, o que é particularmente útil nos vasos torácicos e abdominais, por eliminar os artefatos respiratórios. O tempo de aquisição deve ser curto, não só para ser realizado em uma única apnéia, mas também devido ao comportamento fisiológico do meio de contraste no sistema 
vascular, com o realce venoso iniciando-se apenas alguns segundos após o realce arterial $^{(\mathbf{1 0})}$, podendo dificultar a identificação dos segmentos arteriais pela sobreposição de estruturas venosas.

Além da curta duração, esse tipo de seqüência é ideal para a angio-RM, em virtude da sua alta resolução espacial, alta relação sinal-ruído e por poder ser orientada e reformatada em qualquer plano desejado, devido ao formato volumétrico tridimensional de aquisição, com voxels isotró$\operatorname{picos}^{(\mathbf{1})}$. A vantagem da utilização de unidades isotrópicas de volume está em evitar distorções de imagens reconstruídas em planos diferentes daquele obtido na aquisição dos dados.

Apesar das vantagens da técnica GE ultra-rápida, há um sinal residual persistente nos tecidos perivasculares que pode ser justificado pelo baixo tempo de relaxamento T1 do tecido gorduroso. Para minimizar este efeito, podem-se utilizar técnicas de subtração, em que a imagem com contraste é sobreposta e subtraída da imagem précontraste (Figura 4). O sucesso da técnica de subtração está na perfeita sobreposição das fases pré e pós-contraste, o que é facilmente obtido no crânio ou extremidades, mas de difícil execução na região tóraco-abdominal, devido à manobra respiratória e peristalse ${ }^{(\mathbf{1 3})}$.

Outro artifício potencialmente útil nas sequiências de angio-RM é a supressão de gordura. O principal problema para a utilização desta técnica na angio-RM é o considerável aumento no tempo de aquisição

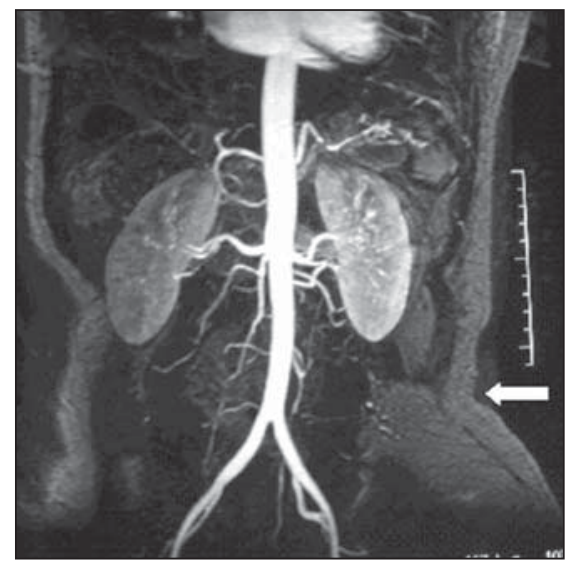

A

Figura 4. A: Imagem angiográfica reconstruída sem a técnica de subtração. Notar o sinal persistente do tecido gorduroso (seta). B: Mesma imagem apresentada em A, reconstruída com a técnica de subtração. Houve apagamento completo do sinal do tecido gorduroso. da seqüência, impeditivo para um exame planejado em apnéia. Este problema pode ser resolvido por técnicas de saturação parcial da gordura, desenvolvidas inicialmente para a angio-RM do crânio e aplicadas com essa finalidade no abdome a partir de $2000^{(\mathbf{1 3 )}}$. A saturação parcial da gordura consiste na utilização de um pulso de supressão espectral seletiva para a gordura apenas na aquisição do espaço k central, aumentando em apenas alguns segundos o tempo total da sequiência.

Com o objetivo de se obter aquisições volumétricas mais rápidas e com melhor definição, recentemente foi desenvolvido um recurso de mapeamento da matriz prévio à aquisição dos dados, que reduz os passos de decodificação de fase da seqüência. Este recurso tecnológico recebeu a denominação SENSE ("sensitivity encoding”). Além de reduzir em muito o tempo de aquisição (em até um terço do tempo original da sequiência), determina importante melhora da resolução espacial da imagem, permitindo a obtenção de cortes ainda mais finos sem redução significativa da relação sinal/ruído ${ }^{(\mathbf{1 4 - 1 6 )}}$. Além disso, o SENSE reduz a perda de sinal por efeitos de suscetibilidade magnética, que podem degradar a qualidade da imagem ${ }^{(\mathbf{1 5})}$.

\section{CONSIDERAÇÕES SOBRE O ESPAÇO k (FOURIER)}

A aquisição de imagens pela RM difere de todos os outros métodos diagnósticos por utilizar-se de uma plataforma abs-

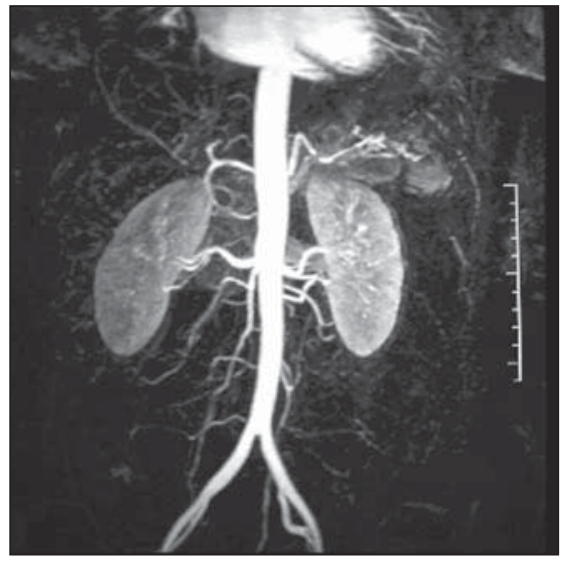

B trata, em que os dados são adquiridos, organizados e então transformados e apresentados na forma de informação visual. Esta plataforma, de compreensão intuitiva, é chamada de espaço k. A escolha da letra k utilizada em sua denominação é decorrente da convenção histórica de físicos e cientistas em denominar as coordenadas espaciais com esta letra ${ }^{(17)}$.

A construção matemática que define o espaço k é complexa e baseada no conceito de um algoritmo denominado "transformada de Fourier", uma série de equações desenvolvidas ainda no início do século 19 para entendimento de princípios físicos da transferência de calor. Fundamentalmente, este algoritmo matemático pode ser aplicado em informações disponíveis em diferentes orientações espaciais para gerar informações em outro plano, como dados bidimensionais $^{(\mathbf{1 7})}$.

Algumas considerações devem ser feitas na compreensão do espaço k: a primeira é que não existe uma correlação direta entre cada ponto do espaço $\mathrm{k}$ e a imagem reconstruída. Na verdade, cada ponto na imagem final recebe, para a sua formação, contribuições de todos os pontos representados no espaço k. Além disso, a maneira que cada ponto no espaço k contribui para o estabelecimento de cada ponto da imagem final é diferente, e depende de onde ele está localizado no espaço k. Especificamente, os pontos da periferia do espaço $\mathrm{k}$ (correspondentes aos dados de alta freqüência espacial) contribuem principalmente para o estabelecimento da resolução dos diversos pontos da imagem. Já os pontos que compõem o espaço k central carregam informações relacionadas ao contraste (relação claro/escuro) de cada ponto da imagem ${ }^{(6,17,18)}$. Portanto, a localização de uma informação no espaço k determina sua contribuição na resolução ou contraste da imagem, e não uma representação direta voxel $\times$ pixel como o hábito radiológico intuitivo.

A construção da imagem por RM é um processo de duas etapas: primeiramente, informações adquiridas são distribuídas num plano abstrato, chamado espaço k, sendo a seguir manipuladas por um algoritmo (transformada de Fourier) para gerar a imagem final. Pelas características desse algoritmo, todos os pontos que compõem 
o espaço k contribuem para a formação de cada ponto da imagem final, alguns com maior contribuição na resolução da imagem (espaço k periférico) e outros mais importantes no estabelecimento do contraste (espaço k central).

A primeira parte desse processo, ou seja, a construção do espaço k, pode ser feita de maneira linear ou priorizando a região central, neste caso maximizando o contraste da imagem.

Tradicionalmente, para a aquisição do espaço k, a decodificação de fase era feita de maneira linear e seqüencial ao longo da área em estudo, partindo-se dos dados de freqüência espacial negativa e finalizando com o componente de frequiência espacial positiva, de modo que o espaço k central era adquirido na metade do processo ${ }^{(9)}$. Com o objetivo de priorizar a aquisição do espaço k central, foram desenvolvidas técnicas de aquisição que obtêm o espaço $\mathrm{k}$ central nos primeiros segundos da seqüência, período de pico da curva de realce vascular, conhecidas como aquisição espiral ou randomizada central ${ }^{(6,19)}$, esta última denominada, por alguns fabricantes, de CENTRA. Por este método, prioriza-se o contraste da imagem.

A aquisição do espaço k de padrão espiral ou randomizado central é vantajosa e justificada pelo fato de garantir o adequado contraste da imagem, mesmo com a interrupção da apnéia, evitando-se também a contaminação do sinal proveniente do sistema venoso, contrastado tardiamente, o que é particularmente útil nos estudos por angio- $\mathrm{RM}^{(\mathbf{6 , 1 9})}$.

\section{CONSIDERAÇÕES TEMPORAIS}

Devido às características farmacocinéticas do gadolínio, a angio-RM com contraste constitui um método dinâmico e imediato, devendo ser realizada na primeira passagem vascular do meio de contraste para um adequado estudo arterial. Imagens posteriores podem ser obtidas se o objetivo for a avaliação de estruturas venosas, porém com a contaminação da imagem decorrente da recirculação do contraste. Neste caso, as partes moles já não terão o sinal totalmente suprimido, devido à difusão intersticial do gadolínio, com o conseqüente incremento no ruído da imagem ${ }^{(\mathbf{1 0 , 2 0})}$.
Independentemente da orientação da decodificação de fase utilizada, o tempo de aquisição dos dados deve ser ajustado de acordo com a injeção do meio de contraste, de modo que a duração da sequiência coincida com o período de passagem da coluna de contraste pelo território vascular de interesse. Como a intensidade de sinal vascular é determinada pela concentração do gadolínio no período em que o centro do espaço k é adquirido, a adequação desse momento tem enorme influência no contraste da imagem resultante ${ }^{(\mathbf{2 0})}$.

O planejamento correto do tempo de início da aquisição dos dados visa obter o máximo de sinal arterial com o mínimo de sinal venoso, lembrando que o pico do sinal arterial ocorre apenas cerca de oito a dez segundos antes do início da contrastação venosa.

Se a aquisição do espaço k central for erroneamente precoce, a imagem apresentará artefatos "leading edge", relacionados à variação abrupta da concentração de gadolínio quando o bolo está chegando à área vascular de interesse (Figura 5). Na prática, estes artefatos resultam em aparente aumento do calibre vascular, com contornos lineares paralelos adjacentes às paredes vasculares, que não devem ser confundidos com artefatos fantasmas de movimento. Artefatos como esses serão mais intensos quando o centro do espaço $\mathrm{k}$ for adquirido antes do pico de concentração do gadolínio $^{(6)}$. Se a aquisição for retardada, a conseqüência será uma intensidade de sinal arterial reduzida, associada à indesejável contaminação da imagem pelo realce venoso.

Para aumentar a complexidade do planejamento temporal, o tempo decorrido entre a injeção do meio de contraste na veia periférica até a contrastação das artérias de interesse é extremamente variável entre diferentes indivíduos ${ }^{(\mathbf{1 0})}$

A princípio, com o objetivo de sincronizar o tempo de injeção à aquisição da imagem, utilizava-se a técnica do melhor palpite ("best guess"), método subjetivo e impreciso, em que o tempo de percurso do meio de contraste era estimado considerando-se a idade, história clínica e função cardíaca de cada paciente. Por sua alta imprecisão, foi sendo progressivamente substituído por outros métodos. Atualmente, po-

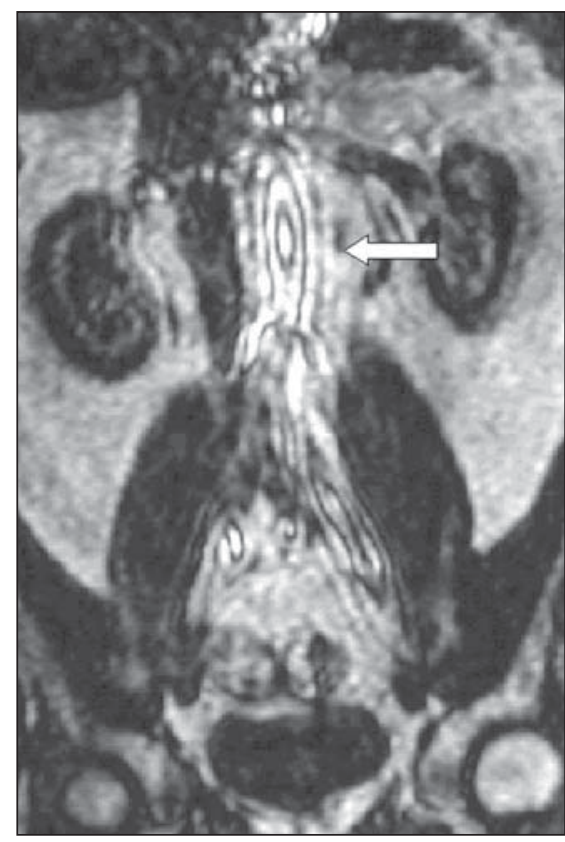

Figura 5. Artefato de "leading edge" devido à aquisição precoce das imagens. A rápida variação na concentração do contraste e no sinal intravascular causa aparente aumento do calibre vascular e multiplicação dos contornos do vaso sanguíneo (seta).

dem ser utilizadas as seguintes técnicas para planejamento do intervalo de tempo do início da aquisição ${ }^{(2,21)}$ :

\section{a) Dose teste}

Por este método estuda-se o tempo necessário para a chegada do bolo de contraste da punção venosa até o território arterial em questão. Isto é feito através da administração de pequena dose de contraste (cerca de 1 a $2 \mathrm{ml}$ de gadolínio seguido por $15 \mathrm{ml}$ de solução salina) e monitorização contínua da intensidade do sinal vascular na região de interesse (Figura 6). Conhecendo-se este intervalo de tempo em cada paciente, a aquisição das imagens pode ser planejada exatamente no período de maior realce do sinal arterial, por intermédio da seguinte fórmula ${ }^{(22)}$ :

$$
\mathrm{T}_{\text {intervalo }}=\mathrm{T}_{\text {circ }}+\mathrm{T}_{\text {gad }} / 2-\mathrm{T}_{\text {aquis }} / 2
$$

onde: $\mathrm{T}_{\text {intervalo }}$ corresponde ao tempo de retardo que se procura para iniciar a sequiência após a injeção do contraste endovenoso; $\mathrm{T}_{\text {circ }}$ corresponde ao tempo de percurso do bolo de contraste, obtido pela seqüência da dose teste; $\mathrm{T}_{\text {gad }}$ corresponde à duração da injeção de gadolínio; $\mathrm{T}_{\text {aquis }}$ corresponde ao tempo de aquisição. 


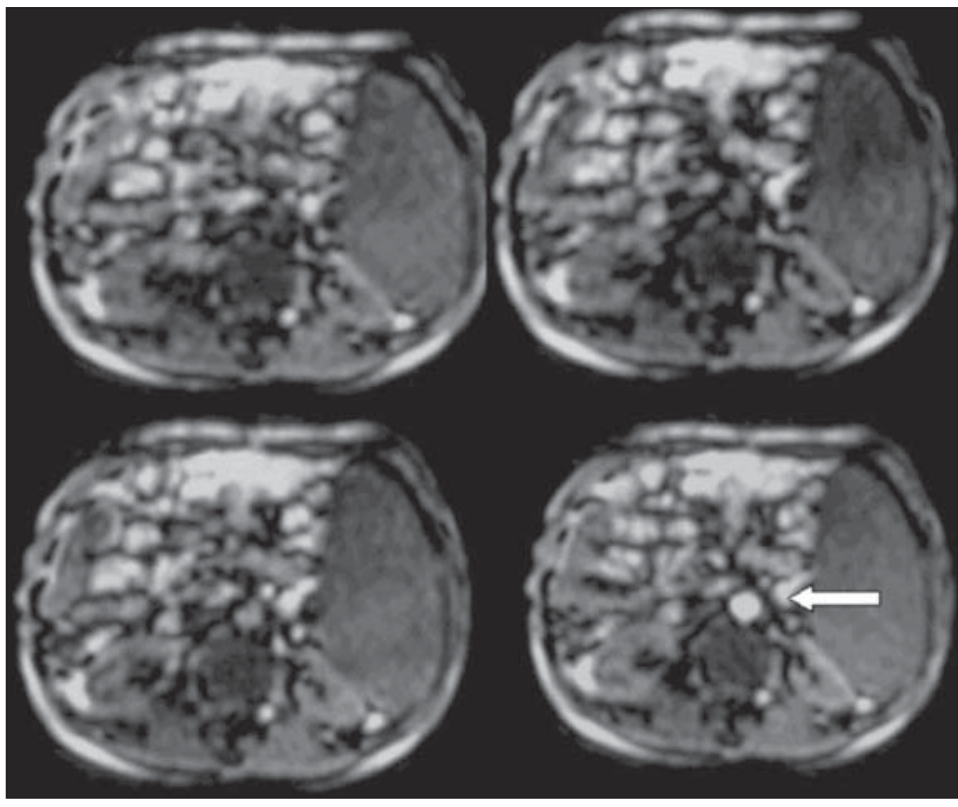

Figura 6. Imagens seqüenciais de rápida aquisição ponderadas em T1, obtidas após a injeção endovenosa de $1 \mathrm{ml}$ de gadolínio, para determinação do tempo de percurso do bolo até o território vascular de interesse (dose teste). A seta aponta a contrastação da aorta no momento de chegada do bolo de contraste.

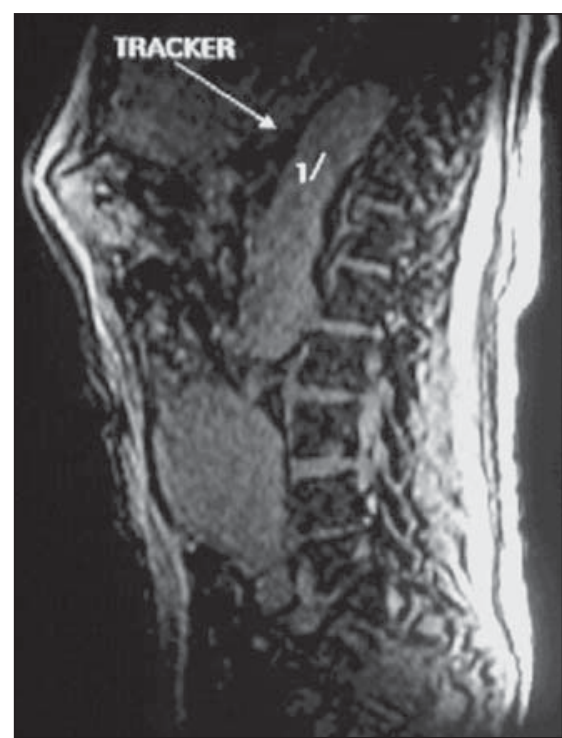

Figura 7. Demonstração do planejamento da técnica de detecção automática do bolo de contraste (“SmartPrep"). 0 rastreador do contraste (identificado como "tracker") foi posicionado no trecho proximal da aorta abdominal.

\section{b) Detecção automática do bolo de contraste ("SmartPrep")}

Nesta técnica usa-se um mecanismo automático de monitorização do sinal vascular em determinada área estabelecida (Figura 7). A chegada do gadolínio na região de interesse produz rápido aumento do sinal vascular, sendo automaticamente desencadeado o início da seqüência após pequena pausa para a inspiração que antecede a apnéia ${ }^{(23,24)}$.

\section{c) Gatilho fluoroscópico ("bolus track")}

Uma série de sequiências bidimensionais ultra-rápidas permite a visualização dos vasos de interesse em tempo real, podendo-se iniciar a aquisição tridimensional assim que o meio de contraste atingir a região (Figura 8). Não há a necessidade da dose teste, e a sobreposição do sinal venoso é mínima porque a decodificação de fase utilizada, de orientação elíptica/central, fornece um contraste de imagem predominantemente arterial $^{(25)}$.

\section{d) Aquisição da imagem em múltiplas fases}

Por este método, o tempo de aquisição é curto o suficiente para tornar possível a obtenção de imagens sucessivas durante a

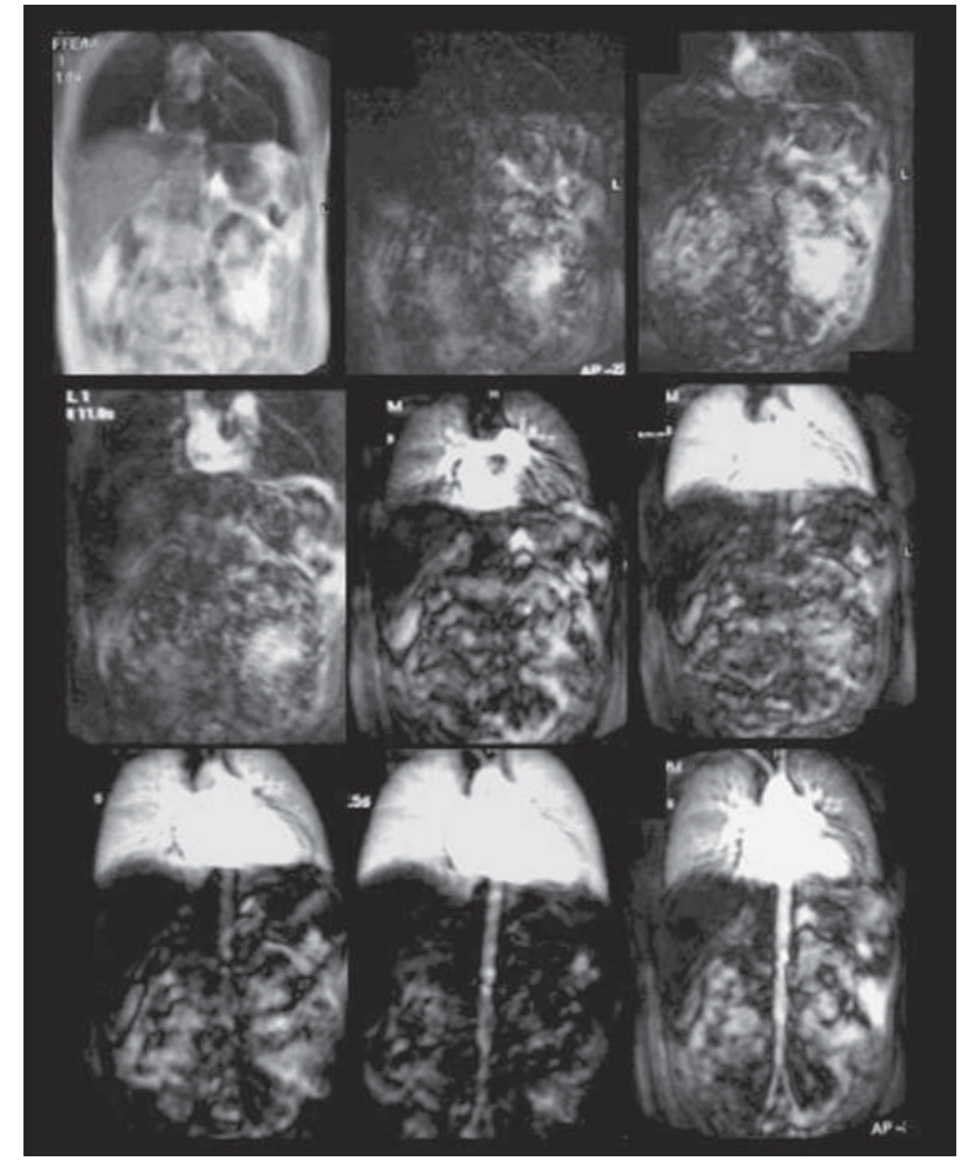

Figura 8. Imagens seriadas de seqüência dinâmica 2D ponderada em T1 obtida em tempo real ("bolus track"). Por esta técnica, é possível acompanhar a progressão do bolo de contraste injetado e iniciar a seqüência angiográfica 3D no momento em que o território vascular de interesse é atingido (última imagem à direita). 
injeção de contraste. A aquisição dos dados e a injeção do contraste são iniciadas simultaneamente, sem conhecimento prévio do tempo de chegada do bolo à região em estudo. Como múltiplos blocos tridimensionais consecutivos são obtidos, há sempre um deles com adequado realce arterial. Por outro lado, esta técnica envolve maior suscetibilidade a artefatos pelos processos de pós-processamento, considerando-se que em busca da maior rapidez, prioriza-se a aquisição do espaço $\mathrm{k}$ central (contraste), com menor freqüência de aquisição das linhas do espaço k periférico (resolução) ${ }^{(21)}$. Apesar dessa aparente deficiência técnica, estudos têm mostrado que a aquisição assimétrica do espaço k utilizada não causa degradação significativa na qualidade da imagem, com resultados comparáveis aos obtidos em sequiências de angio-RM com a técnica contrastada habitual ${ }^{(21,26)}$. Este método somente é possível na presença de gradientes de alta "performance", que permitem o uso de TR e TE extremamente curtos (inferiores a $4 \mathrm{~ms}$ e $2 \mathrm{~ms}$, respectivamente) ${ }^{(\mathbf{2 1})}$.

Todas essas técnicas procuram garantir que o pico de sinal arterial e a aquisição do espaço k central ocorram simultaneamente, cada uma apresentando vantagens e desvantagens entre si. Por exemplo, a técnica de aquisição multifase, que dispensa qualquer planejamento temporal, implica relativa redução da resolução espacial. Já a técnica da dose teste tem resolução espacial maior que esta primeira, no entanto apresenta maior ruído de imagem, devido ao elevado sinal tecidual relacionado à rápida redistribuição da dose teste pelo espaço intersticial, além da contrastação das vias coletoras renais, mesmo em doses mínimas de contraste administrado (Figura 9). A presença de contraste no sistema pielo-calicinal pode ser um importante fator de limitação na avaliação das artérias renais $^{(21)}$. Na detecção automática do contraste, os principais erros estão relacionados ao mau posicionamento da área de medida do sinal vascular e à movimentação do paciente, com conseqüente perda da posição estabelecida inicialmente. Já o gatilho fluoroscópico oferece alta resolução espacial sem o uso da dose teste, constituindo uma das melhores alternativas disponíveis atualmente ${ }^{(26)}$.

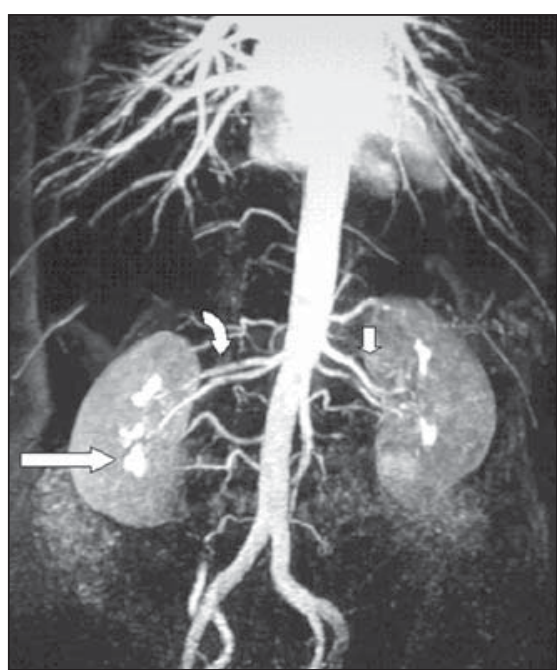

Figura 9. Reconstrução MIP no plano coronal em exame realizado com a técnica da dose teste. Notar a contrastação dos cálices renais, mesmo com a utilização de apenas $1 \mathrm{ml}$ de gadolínio como dose teste (seta). Há duplicidade da artéria renal esquerda (seta pequena) e bifurcação precoce da artéria renal direita (seta curva), variantes da normalidade.

\section{MEIO DE CONTRASTE PARAMAGNÉTICO (GADOLÍNIO)}

O gadolínio $\left(\mathrm{Gd}^{3+}\right)$ é um íon metálico paramagnético que reduz tanto o tempo de relaxamento T1 (spin-meio) como o tempo de relaxamento T2 (spin-spin). Devido à toxicidade biológica de sua forma iônica, ele é usado como um quelato, geralmente ligado ao DTPA numa solução iônica bastante estável e de raros efeitos adversos ${ }^{(\mathbf{1 8})}$.

No organismo, o gadolínio tem comportamento farmacológico semelhante ao meio de contraste iodado, ou seja, atua como um agente extracelular, difundindose rapidamente do compartimento intravascular para o espaço intersticial ${ }^{(\mathbf{1 8})}$. Acredita-se que cerca de $80 \%$ da dose deixem o compartimento vascular nos cinco primeiros minutos após a injeção. Por esse motivo, a aquisição das imagens na angio-RM contrastada deve ser rápida. O gadolínio não entra nas células nem é metabolizado pelo organismo, sendo excretado por filtração glomerular, com meia-vida biológica de aproximadamente 90 minutos $^{(27)}$.

A segurança é a mais importante vantagem do contraste paramagnético. Comparado ao contraste iodado utilizado em estudos radiológicos, o gadolínio tem freqüência bastante reduzida de complicações ou reações adversas, além de mínima nefrotoxicidade, mesmo nas doses necessárias para a angio-RM ${ }^{(\mathbf{2 8 , 2 9 )}}$. Além disso, o pequeno volume injetado evita os efeitos relacionados à alta carga osmolar, como ocorre com o contraste iodado ${ }^{(29)}$.

Reações adversas são raras com o gadolínio, ocorrendo em cerca de 1-2\% das exposições, e na maioria dos casos correspondem a queixas inespecíficas e autolimitadas, como náuseas e cefaléia ${ }^{(28,29)}$. Em estudo duplo-cego realizado na época da regulamentação do uso do gadolínio endovenoso nos EUA, a frequiência de reações adversas em dois grupos de pacientes, um deles submetido ao gadolínio e o outro à solução salina, foi semelhante ${ }^{(\mathbf{3 0})}$. Reações alérgicas graves ao gadolínio são casos de exceção, com descrições isoladas e esporádicas na literatura de reações anafilatóides $^{(28)}$. A segurança da forma rápida em bolo de administração do gadolínio também já foi testada, sem aumento na frequiência de reações adversas ${ }^{(\mathbf{2 8})}$.

Se por um lado o gadolínio tem custo mais alto que o contraste iodado, todo o procedimento diagnóstico da angio-RM contrastada ainda é consideravelmente mais barato que a $\operatorname{arteriografia}^{(31)}$.

\section{Dose do meio de contraste}

A dose do meio de contraste paramagnético deve ser suficiente para reduzir o tempo de relaxamento T1 do sangue arterial a um nível inferior ao de qualquer outro tecido, fornecendo elevado contraste de imagem e alta razão sinal/ruído. $\mathrm{O}$ tecido com mais baixo T1 no organismo é a gordura, correspondendo a cerca de 270 ms em campo magnético de 1,5 T. Portanto, a dose de gadolínio deve ser suficiente para reduzir o T1 do sangue a um nível mais baixo que esse valor ${ }^{(\mathbf{1 8})}$. Para atingir tal objetivo, a dose de gadolínio deve ser igual ou superior a $0,1 \mathrm{mmol} / \mathrm{kg}$, sendo freqüentemente utilizada na forma de dose dupla $(0,2 \mathrm{mmol} / \mathrm{kg})$ para a obtenção de uma coluna de contraste mais persistente e homogênea ${ }^{(\mathbf{1 8 , 3 2 , 3 3 )}}$. No entanto, há evidências recentes de que é possível reduzir a dose, com bons resultados ${ }^{(34)}$. Com o progressivo desenvolvimento dos recursos técnicos e o conseqüente ganho na resolução temporal e espacial das sequiências de exame (relacionadas, respectivamente, à velocidade de aquisição e à qualidade da 
imagem), começou a ser investigada a eficácia da dose simples de contraste na angio-RM quando comparada à dose dupla, principalmente considerando-se a economia relacionada ao alto custo do contraste paramagnético ${ }^{(35)}$. Com este intuito foram realizados estudos em alguns territórios arteriais com bons resultados a favor da dose simples de gadolínio, como na avaliação da aorta abdominal ${ }^{(34,35)}$, artérias renais ${ }^{(22,33,36)}$ e artérias carótidas ${ }^{(37)}$

Pesquisas no sentido de otimizar a injeção do contraste, visando à restrição de sua dose sem prejuízo para a qualidade de imagem, são fundamentais no planejamento de procedimentos terapêuticos endoluminais guiados pela angio-RM, por motivos tanto farmacológicos de hiperdosagem como econômicos ${ }^{(\mathbf{1 2})}$. Parece ser este o principal caminho em que segue a investigação da literatura na área.

\section{TÉCNICA DE INJEÇÃO}

Conceitualmente, a velocidade de injeção ideal na angio-RM é aquela que garante a presença homogênea do meio de contraste no território arterial sob investigação, durante a aquisição do espaço $\mathrm{k}$ central. Com a aquisição do espaço $\mathrm{k}$ de padrão central, a prioridade deve ser dada em obter um bolo mais compacto (utilizando maior velocidade de injeção), de modo que o platô de realce arterial seja mais curto e concomitante com a aquisição do espaço $\mathrm{k} \mathrm{central}^{(38)}$.

Sabe-se que a intensidade do sinal vascular aumenta proporcionalmente à velocidade de injeção, até o valor de 1,5 a 2 $\mathrm{ml} / \mathrm{s}$. Por outro lado, dados da literatura reforçam que a dose dupla de contraste $(0,2$ $\mathrm{mmol} / \mathrm{kg}$ ) administrada a uma velocidade de 2 a $3 \mathrm{ml} / \mathrm{s}$ apresenta bons resultados na grande maioria dos pacientes ${ }^{(\mathbf{1 8})}$.

A injeção deve ser feita por meio de bomba mecânica, por oferecer um fluxo mais constante e padronizado ${ }^{(39)}$. Porém, muitas instituições não dispõem de bombas mecânicas e apresentam bons resultados com injeção manual em mãos experientes. De qualquer modo, todo bolo injetado deve ser seguido por um volume de 15 a $20 \mathrm{ml}$ de solução salina, para garantir a entrada vascular de todo o meio de contraste e aumentar o fluxo venoso do braço após a injeção.

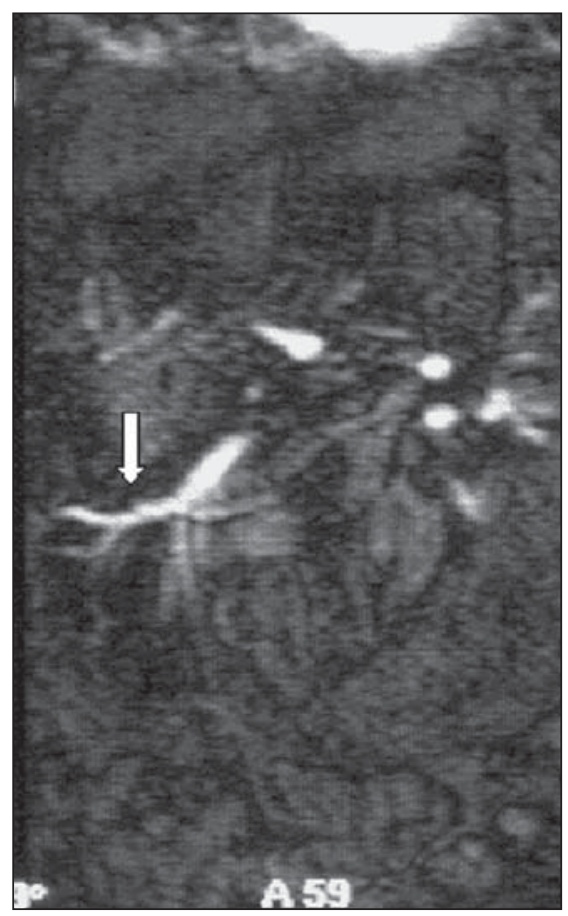

B

\section{PLANOS DE AQUISIÇÃO}

A sequiência dinâmica da angio-RM com contraste pode ser adquirida em qualquer plano espacial. Preferencialmente, deve-se optar pelo planejamento da seqüência no plano de maior orientação dos vasos em estudo, de modo a facilitar a interpretação das imagens originais.

\section{ESTUDO EM VÁRIAS FASES VASCULARES}

Como se discutiu previamente, a ênfase do estudo é dada à adequada aquisição da fase arterial de exame, com repetição da sequiência nas fases venosa e de equilíbrio (Figura 10). As fases tardias podem ser úteis para avaliar estruturas venosas, o padrão de realce de órgãos parenquimatosos ou até avaliar a função glomerular renal e a anatomia das vias excretoras.

$\mathrm{Na}$ angio-RM do abdome, por exemplo, com duração da aquisição na fase arterial de aproximadamente 20 segundos, podese esperar cerca de 30 a 40 segundos e iniciar uma nova aquisição de imagens. Este tempo (50 a 60 segundos após o início da

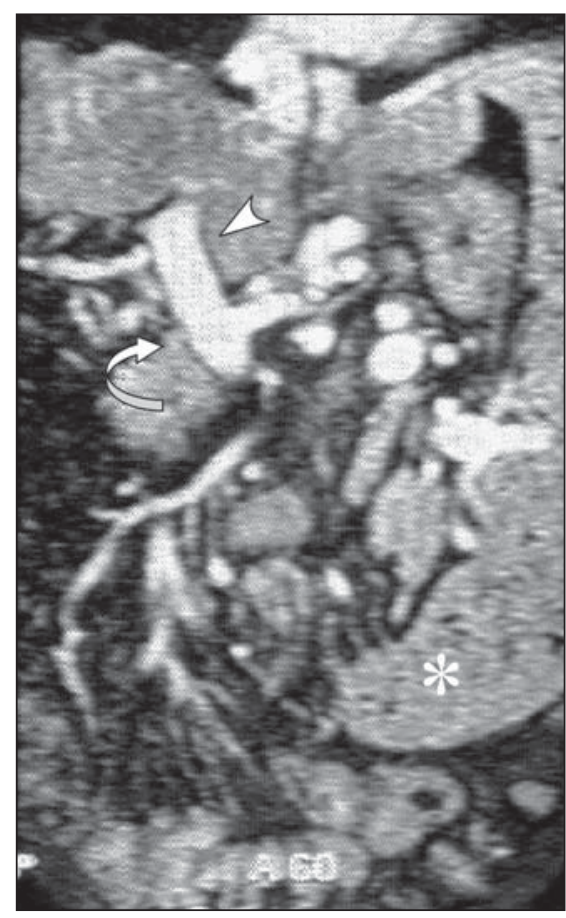

C

Figura 10. Exemplo de imagens originais da seqüência coronal dinâmica FFE nas fases pré-contraste, arterial e venosa. A: Fase pré-contraste obtida como máscara de subtração. B: Fase arterial demonstrando o terço distal e ramificações terminais da artéria mesentérica superior (seta). C: Fase venosa portal no mesmo plano das imagens A e B, com a contrastação da veia porta (cabeça de seta) e junção espleno-portal (seta curva). Notar a esplenomegalia volumosa (asterisco), responsável pelo deslocamento da artéria mesentérica superior para a direita. 
injeção) é suficiente para avaliar o realce da veia porta e a perfusão de órgãos parenquimatosos.

\section{PROCESSAMENTO E APRESENTAÇÃO DAS IMAGENS}

As seqüências tridimensionais de angioRM fornecem imagens na forma de dados volumétricos, sendo necessária a reformatação em múltiplos cortes finos para apresentação nos planos axial, sagital ou coronal. Além disso, a manipulação posterior dos dados na estação de trabalho permite eliminar estruturas sobrepostas ou desdobrar vasos tortuosos.

Entre as técnicas de pós-processamento disponíveis, destacam-se a projeção de intensidade máxima (MIP - "maximum intensity projection"), a representação de superfície e a endoscopia intraluminal virtual (angioscopia virtual) ${ }^{(\mathbf{1 1}, \mathbf{4 0 , 4 1 )}}$.

A técnica MIP consiste na representação bidimensional dos voxels de maior sinal em determinada projeção escolhida, sendo obtida por algoritmos de projeção de raios. É técnica rápida, de fácil execução, fornecendo imagens muito semelhantes às obtidas na angiografia convencional ${ }^{(41)}$. As reconstruções vasculares com técnica MIP também não estão livres de artefatos, destacando-se, nesta fase do processo, os artefatos "em escada", que correspondem a irregularidades das paredes vasculares com aspecto em pequenos degraus. Estes artefatos são geralmente decorrentes da utilização de cortes espessos na reconstrução das imagens originais ou aumento do "gap" entre os cortes obtidos (Figura 11).

A representação de superfície apresenta a imagem angiográfica em perspectiva tridimensional. É útil nas alterações que deformam a superfície vascular ou em alterações morfológicas ${ }^{(\mathbf{4 1 )}}$.

Já na angioscopia virtual o observador é posicionado no interior do vaso, com representação em perspectiva tridimensional da visão intraluminal. Exige maior tempo de processamento e interpretação das imagens, sendo útil na avaliação de lesões da parede vascular interna como trombos, e na estimativa de estenoses ${ }^{(\mathbf{4 1}, \mathbf{4 2})}$.

Apesar dos excelentes angiogramas obtidos com as técnicas de pós-processamento, as reconstruções finas multiplanares

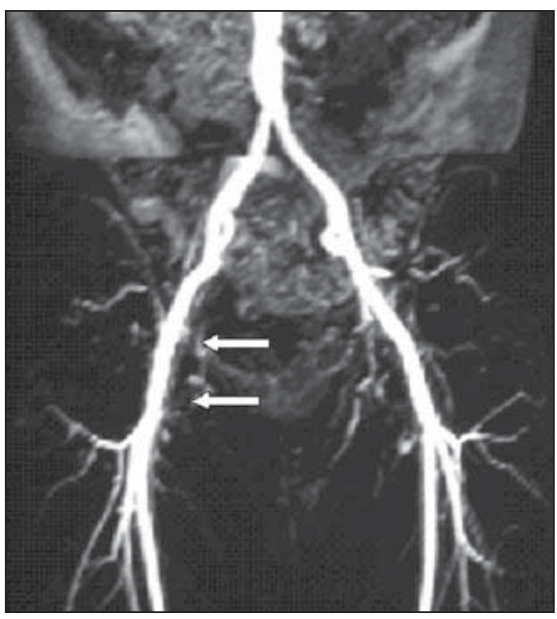

Figura 11. A: Artefatos de reconstrução "em escada" em imagens reconstruídas com técnica MIP nas artérias femorais comuns (setas). B: Detalhe da artéria femoral comum direita mostrada em $\mathbf{A}$.

originais não devem ser desprezadas, pois informações diagnósticas importantes podem ser suprimidas no processo de manipulação dos dados. A maioria das alterações vasculares deve ser estudada utilizando-se angiogramas por técnica MIP como orientação ou mapa de leitura, baseandose a impressão diagnóstica principalmente nas imagens originais ${ }^{(\mathbf{1 1})}$

Outra opção para o estudo vascular na forma de pós-processamento está na reformatação das imagens dinâmicas planejadas habitualmente no estudo abdominal, de modo a fornecer imagens com a apresentação de um angiograma ${ }^{(\mathbf{4 3})}$. Em trabalho publicado em 2000, houve adequada visualização dos maiores troncos arteriais, demonstrando a versatilidade da técnica de RM na manipulação das imagens ${ }^{(\mathbf{4 3})}$.

\section{PERSPECTIVAS FUTURAS}

Entre os principais focos de atenção da literatura atual em angio-RM contrastada destacam-se a angio-RM da aorta e artérias dos membros inferiores com movimentação da mesa ("Mobitrack") ${ }^{(44)}$, a angio-RM de corpo inteiro (angioSURF) ${ }^{(\mathbf{4 5})}$, as técnicas de angio-RM em tempo real (angiofluoroscopia por RM) ${ }^{(46)}$ e os novos contrastes endovenosos, com destaque aos contrastes de micropartículas de óxido de ferro superparamagnético ${ }^{(47)}$.

A princípio, a avaliação de segmentos arteriais extensos como os membros inferiores representavam um desafio à parte para a angio-RM com contraste. Atualmente, a aorta e as artérias dos membros inferiores podem ser estudadas por uma metodologia já amplamente comprovada e disponível para este território, que combina a injeção endovenosa do gadolínio a imagens seqüenciais obtidas consecutivamente em três leitos arteriais (aorto-ilíaco, fêmoro-poplíteo e poplíteo-tíbio-fibular), numa tecnologia sincronizada denominada, em alguns modelos, como "Mobitrack" (Figura 12). Esta técnica de estudo tem apresentado elevada acurácia em comparação à angiografia para o diagnóstico de doença artério-oclusiva ${ }^{(44)}$

A angio-RM de corpo inteiro foi desenvolvida a partir de 2000 através de um sistema de bobinas e movimentação coordenada da mesa chamado angioSURF, proposto como método de rastreamento para doenças vasculares difusas ${ }^{(45)}$. Permite a visualização de praticamente todo o sistema arterial, das artérias carótidas às artérias tibiais, porém ainda não constitui um método estabelecido quanto à sua aplicabilidade, tampouco amplamente disponível, ainda permanecendo restrito aos maiores centros de desenvolvimento tecnológico.

A possibilidade de executar procedimentos endoluminais por cateteres guiados pela RM gerou experimentações nesse sentido, e já é possível executar seqüências geradas e apresentadas em tempo real, com adequada qualidade da imagem ${ }^{(\mathbf{4 6})}$. $\mathrm{O}$ fator que passa então a ser restritivo é a superdosagem do gadolínio. Para solucionar este 


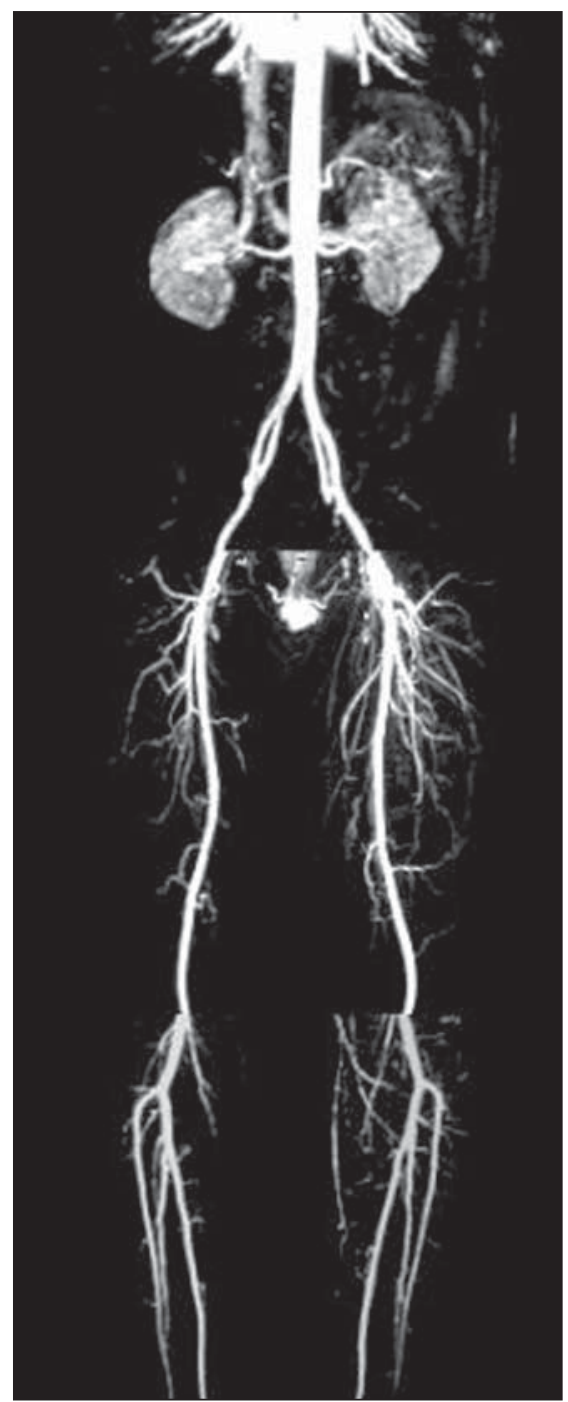

Figura 12. Reconstrução MIP de exame realizado com a técnica "Mobitrack" para estudo da aorta abdominal e artérias dos membros inferiores.

problema, a literatura tem apresentado como alternativa os meios de contraste de prolongada permanência vascular, por meio da combinação do gadolínio com proteínas plasmáticas, ou pelo uso de micropartículas de óxido de ferro superparamagnético, que não serão excretados por filtração glomerular, e sim por depuração pelo sistema retículo-endotelial. Esses últimos materiais ainda não foram aprovados para uso clínico em humanos, com os estudos publicados até o momento ainda experimentais, descrevendo sua aplicabilidade em cobaias $^{(\mathbf{4 7}, 48)}$.

Entre os novos contrastes endovenosos recentemente incorporados para estudos vasculares pela angio-RM destacam-se dois grupos: os contrastes de baixa ligação com proteínas plasmáticas, cujo melhor exemplo é o gadobenato de dimeglumina, e os contrastes de micropartículas. O gadobenato de dimeglumina determina maior intensidade de sinal vascular que o gadopentetato de dimeglumina habitualmente usado, para a mesma dose e velocidade de fluxo $^{(\mathbf{3 3})}$. Este efeito é determinado pela maior fração livre do contraste sérico. Entretanto, já se demonstrou que, apesar da maior intensidade de sinal vascular, não há diferença significante da relação sinal-ruído e freqüência de detecção de lesões entre os dois meios de contraste, testada no leito arterial renal ${ }^{(33)}$.

\section{CONCLUSÃO}

O papel atual da angio-RM como ferramenta diagnóstica merece destaque na avaliação de anomalias anatômicas, estenoses, oclusões e complicações vasculares póscirúrgicas, principalmente nos casos de transplantes de órgãos ${ }^{(\mathbf{3 , 8}, \mathbf{4 9 - 5 1 )}}$. Suas principais vantagens estão na não utilização do contraste iodado ou radiação ionizante, rapidez e fácil execução, mínima invasividade e possibilidade de avaliar complementarmente o parênquima de órgãos adjacentes de interesse diagnóstico ${ }^{(\mathbf{5 0}, 52)}$.

\section{REFERÊNCIAS}

1. Riederer SJ, Wood ML. Categorical course in physics: the basis physics of MR imaging. In: $83 \mathrm{rd} \mathrm{Sci-}$ entific Assembly and Annual Meeting of the RSNA, Chicago, 1997.

2. Heiss SG, Li KC. Magnetic resonance angiography of mesenteric arteries. A review. Invest Radiol 1998;33:670-81.

3. D'Ippolito G, Wolosker N, Galvão Filho M, Kalil JA, Wolosker A, Borri ML. Angio-RM com contraste no estudo das doenças da aorta tóraco-abdominal. Rev Imagem 1998;20:85-93.

4. Gilfeather M, Holland GA, Siegelman ES, et al. Gadolinium-enhanced ultrafast three-dimensional spoiled gradient-echo MR imaging of the abdominal aorta and visceral and iliac vessels. RadioGraphics 1997; 17:423-32.

5. Prince MR. Gadolinium-enhanced MR aortography. Radiology 1994;191:155-64.

6. Maki JH, Prince MR, Londy FJ, Chenevert TL. The effects of time varying intravascular signal intensity and $\mathrm{k}$-space acquisition order on three-dimensional MR angiography image quality. J Magn Reson Imaging 1996;6:642-51.

7. Debatin JF, Hany TF. MR-based assessment of vascular morphology and function. Eur Radiol 1998; 8:528-39.

8. Laub G. Principles of contrast-enhanced MR angiography. Basic and clinical applications. Magn Reson Imaging Clin N Am 1999; 7:783-95.
9. Prince MR, Narasimham DL, Stanley JC, et al. Breath-hold gadolinium-enhanced MR angiography of the abdominal aorta and its major branches. Radiology 1995;197:785-92.

10. Alley MT, Shifrin RY, Pelc NJ, Herfkens RJ. Ultrafast contrast-enhanced three-dimensional MR angiography: state of the art. RadioGraphics 1998;18: 273-85.

11. Davis CP, Hany TF, Wildermuth S, Schmidt M Debatin JF. Postprocessing techniques for gadolinium-enhanced three-dimensional MR angiography. RadioGraphics 1997; 17:1061-77.

12. Schild HH, Kuhl CK. Contrast-enhanced magnetic resonance angiography. Potential applications and pitfalls in magnetic resonance angiography-guided therapy: a review. Invest Radiol 1998;33:524-7.

13. Hilfiker PR, Herfkens RJ, Heiss SG, Alley MT, Fleischmann D, Pelc NJ. Partial fat-saturated contrast-enhanced three-dimensional MR angiography compared with non-fat-saturated and conventional fat-saturated MR angiography. Radiology 2000; 216:298-303.

14. Weiger M, Pruessmann KP, Kassner A, et al. Contrast-enhanced 3D MRA using SENSE. J Magn Reson Imaging 2000;12:671-7.

15. Weiger M, Pruessmann KP, Osterbauer R, Bornert P, Boesiger P, Jezzard P. Sensitivity-encoded singleshot spiral imaging for reduced susceptibility artifacts in BOLD fMRI. Magn Reson Med 2002;48: 860-6.

16. Maki JH, Wilson GJ, Eubank WB, Hoogeveen RM Utilizing SENSE to achieve lower station sub-millimeter isotropic resolution and minimal venous enhancement in peripheral MR angiography. J Magn Reson Imaging 2002;15:484-91.

17. Mezrich R. A perspective on K-space. Radiology 1995;195:297-315.

18. Maki JH, Chenevert TL, Prince MR. Contrast-enhanced MR angiography. Abdom Imaging 1998; 23:469-84.

19. Willinek WA, Gieseke J, Conrad R, et al. Randomly segmented central k-space ordering in high-spatialresolution contrast-enhanced MR angiography of the supraaortic arteries: initial experience. Radiology 2002;225:583-8.

20. Watanabe Y, Dohke M, Okumura A, et al. Dynamic subtraction contrast-enhanced MR angiography: technique, clinical applications, and pitfalls. RadioGraphics 2000;20:152-3.

21. Schoenberg SO, Bock M, Knopp MV, et al. Renal arteries: optimization of three-dimensional gadolinium-enhanced MR angiography with bolus-timing-independent fast multiphase acquisition in a single breath hold. Radiology 1999;211:667-79.

22. Lee VS, Rofsky NM, Krinsky GA, Stemerman DH, Weinreb JC. Single-dose breath-hold gadoliniumenhanced three-dimensional MR angiography of the renal arteries. Radiology 1999;211:69-78.

23. Prince MR, Chenevert TL, Foo TK, Londy FJ, Ward JS, Maki JH. Contrast-enhanced abdominal MR angiography: optimization of imaging delay time by automating the detection of contrast material arrival in the aorta. Radiology 1997;203:109-14.

24. Ho VB, Foo TK. Optimization of gadolinium-enhanced magnetic resonance angiography using an automated bolus-detection algorithm (MR SmartPrep). Original investigation. Invest Radiol 1998; 33:515-23

25. Wilman AH, Riederer SJ, King BF, Debbins JP, Rossman PJ, Ehman RL. Fluoroscopically triggered contrast-enhanced three-dimensional MR 
angiography with elliptical centric view order: application to the renal arteries. Radiology 1997;205: 137-46.

26. Van Hoe L, De Jaegere T, Bosmans H, et al. Breathhold contrast-enhanced three-dimensional MR angiography of the abdomen: time-resolved imaging versus single-phase imaging. Radiology 2000;214 149-56.

27. Dawson P. Contrast agents in magnetic resonance imaging. Eur J Radiol 1996;23:201-4.

28. Nelson KL, Gifford LM, Lauber-Huber C, Gross CA, Lasser TA. Clinical safety of gadopentetate dimeglumine. Radiology 1995;196:439-43.

29. Niendorf HP, Alhassan A, Geens VR, Clauss W. Safety review of gadopentetate dimeglumine. Extended clinical experience after more than five million applications. Invest Radiol 1994;29 Suppl 2:S179-82.

30. Russell EJ, Schaible TF, Dillon W, et al. Multicenter double-blind placebo-controlled study of gadopentetate dimeglumine as an MR contrast agent: evaluation in patients with cerebral lesions. AJR 1989;152:813-23.

31. Prince MR. Why inject contrast for magnetic resonance angiography? Invest Radiol 1998;33:483-4.

32. Prince MR, Grist TM, Debatin JF. 3D contrast MR angiography. Berlin: Springer-Verlag, 1997.

33. Volk M, Strotzer M, Lenhart M, et al. Renal timeresolved MR angiography: quantitative comparison of gadobenate dimeglumine and gadopentetate dimeglumine with different doses. Radiology 2001; 220:484-8.

34. Thurnher SA, Capelastegui A, Del Olmo FH, et al. Safety and effectiveness of single- versus tripledose gadodiamide injection-enhanced MR angiography of the abdomen: a phase III double-blind multicenter study. Radiology 2001;219:137-46.

35. Lentschig MG, Reimer P, Rausch-Lentschig UL, Allkemper T, Oelerich M, Laub G. Breath-hold gadolinium-enhanced MR angiography of the major vessels at 1.0 T: dose-response findings and angiographic correlation. Radiology 1998;208: 353-7.

36. Korst MB, Joosten FB, Postma CT, Jager GJ, Krabbe JK, Barentsz JO. Accuracy of normal-dose contrast-enhanced MR angiography in assessing renal artery stenosis and accessory renal arteries. AJR 2000;174:629-34.

37. Hartmann L, Wolosker A, D'Ippolito G, Borri M, Kalil J. Angio-RM das artérias carótidas e vertebrais: análise de diferentes técnicas de volume e diluição de contraste em aparelho de 1,0 T e gradiente de 15 mT/m. Radiol Bras 2001;34:201-5.

38. Maki JH, Prince MR, Chenevert TC. Optimizing three-dimensional gadolinium-enhanced magnetic resonance angiography. Original investigation. Invest Radiol 1998;33:528-37.

39. Leung DA, Hany TF, Debatin JF. Three-dimensional contrast-enhanced magnetic resonance angiography of the abdominal arterial system. Cardiovasc Intervent Radiol 1998;21:1-10.

40. Hany TF, Schmidt M, Davis CP, Gohde SC, Debatin JF. Diagnostic impact of four postprocessing techniques in evaluating contrast-enhanced three-dimensional MR angiography. AJR 1998;170:90712.

41. Hernandez-Hoyos M, Orkisz M, Puech P, MansardDesbleds C, Douek P, Magnin IE. Computer-assisted analysis of three-dimensional MR angiograms. RadioGraphics 2002;22:421-36.

42. Glockner JF. Navigating the aorta: MR virtual vascular endoscopy. RadioGraphics 2003;23:e11.

43. Blasbalg R, Mitchell DG, Outwater EK, Ito K, Gabata T, Chiowanich P. Free MRA of the abdomen: postprocessing dynamic gadolinium-enhanced $3 \mathrm{D}$ axial $\mathrm{MR}$ images. Abdom Imaging 2000;25:62-6.

44. Meaney JF, Ridgway JP, Chakraverty S, et al. Step- ping-table gadolinium-enhanced digital subtraction MR angiography of the aorta and lower extremity arteries: preliminary experience. Radiology 1999; 211:59-67.

45. Goyen M, Herborn CU, Lauenstein TC, et al. Optimization of contrast dosage for gadobenate dimeglumine-enhanced high-resolution whole-body 3D magnetic resonance angiography. Invest Radiol 2002;37:263-8.

46. Serfaty JM, Atalar E, Declerck J, et al. Real-time projection MR angiography: feasibility study. Radiology 2000;217:290-5.

47. Wacker FK, Reither K, Ebert W, Wendt M, Lewin JS, Wolf KJ. MR image-guided endovascular procedures with the ultrasmall superparamagnetic iron oxide SH U $555 \mathrm{C}$ as an intravascular contrast agent: study in pigs. Radiology 2003;226:459-64.

48. Allkemper T, Bremer C, Matuszewski L, Ebert W, Reimer P. Contrast-enhanced blood-pool MR angiography with optimized iron oxides: effect of size and dose on vascular contrast enhancement in rabbits. Radiology 2002;223:432-8.

49. Gaa J, Georgi M. Non-invasive imaging of abdominal vascular pathologies. Eur Radiol 1998;8:50716.

50. Rankin SC, Jan W, Koffman CG. Noninvasive imaging of living related kidney donors: evaluation with CT angiography and gadolinium-enhanced MR angiography. AJR 2001;177:349-55.

51. Wolosker N, Nakano L, D'Ippolito G, Rosoky RA, Borri ML, Wolosker AM. Gadolinium magnetic angioresonance in the study of aortoiliac disease. Angiology 2003;54:163-8.

52. Stafford-Johnson DB, Hamilton BH, Dong Q, et al Vascular complications of liver transplantation: evaluation with gadolinium-enhanced MR angiography. Radiology 1998;207:153-60. 